\title{
Remote Status Monitoring of Broadband Seismometer Based on the Mobile Internet
}

\author{
Shixue Lv, Huaizhu Zhang *, Jun Lin, Yun Long, Shuai Yuan \\ College of Instrumentation \& Electrical Engineering \\ Jilin University \\ Changchun, China \\ huaizhuzhang@jlu.edu.cn
}

\begin{abstract}
Application fields of broadband seismometer determine that the settlements of the most instruments locate in remote areas in the wild. Especially as the broadband seismometers of scientific exploration networks and mobility observatory arrays are often unattended, and the field inspections of instruments require a lot of manpower and material costs. To solve these problems, we proposed and designed a remote condition monitoring method of broadband seismometer which bases on the mobile Internet. By SMS (Short Messaging Service) and e-mail in two ways, we have achieved remote status monitoring of broadband seismometer. It provided a solution for monitoring of broadband seismometer which located in remote areas in the wild.
\end{abstract}

Keywords_broadband seismometer; mobile internet; remote Monitoring

\section{INTRODUCTION}

With the development of seismological observation technology, broadband seismometer has a wider frequency range, lower noise, greater dynamic range and other characteristics, that makes it have a greater advantage in obtaining information on the deep structure of the Earth, researching the mechanism of the earthquake and so on. It has more and more widely used in the national earthquake monitoring network, regional earthquake monitoring network, scientific exploration networks, mobility observatory arrays, and other fields. Application fields of broadband seismometer determine that the settlements of the most instruments locate in remote areas in the wild. Especially as the broadband seismometers of scientific exploration networks and mobility observatory arrays are often unattended, and the field inspections of instruments require a lot of manpower and material costs. It has become an urgent problem to be solved to how to achieve low-cost remote status monitoring of broadband seismometer. In this paper, we propose a remote status monitoring method of broadband seismometer based on the mobile Internet.

\section{THEORY AND METHOD}

To be able to use the mobile Internet, mobile communication module was built into the broadband seismometer. By the mobile Internet, we achieved remote status monitoring of broadband seismometer. So far, we have realized a total of two status monitoring methods, SMS (Short Messaging Service) and e-mail.

\section{A. SMS Monitoring Method}

Broadband seismometer received status monitoring order sent by the mobile terminal through the mobile communication module, and returned the status information of instrument to the mobile terminal after parsing status monitoring order (Fig.1).
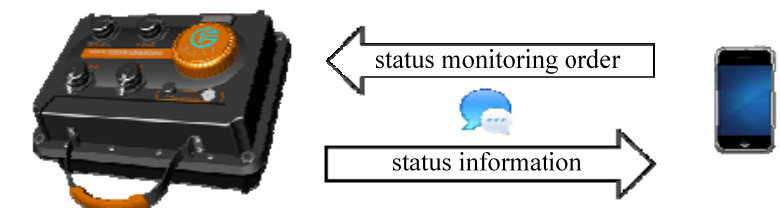

Broadband Seismometer (built-in communication module)

Mobile Terminal

Fig. 1. Schematic diagram of SMS monitoring method.

\section{B. E-mail Monitoring Method}

Broadband seismometer connected to the mobile Internet through the mobile communication module, the status information of instrument was sent in the form of e-mail regularly to the monitoring terminal (Fig.2).

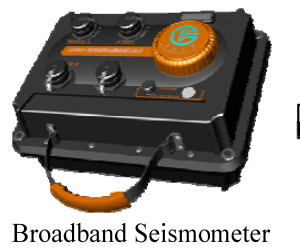

built-in communication module)

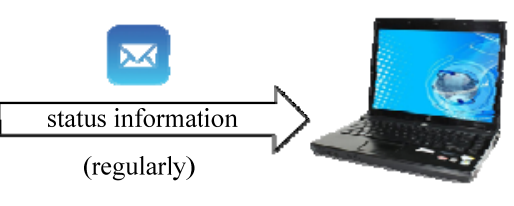

Monitoring Terminal

Fig. 2. Schematic diagram of e-mail monitoring method.

\section{EXAMPLES}

Combined with the project of "Deep Structure Probe of South China" belonged to the Institute of Geology, Chinese Academy of Geological Sciences, We selected 10 stations in south China (Jiangxi, Anhui, Zhejiang) to carry out comparative experiment (ongoing). We used self-developed broadband seismometer with remote status monitoring function, they used Q330S+ produced by Kinemetrics. They need to conduct site inspections to know the status information of instrument, it required a lot of manpower and material costs. 
We could know the status information of instrument by SMS (Fig.3) and e-mail (Fig.4), it was convenient and lowcost.

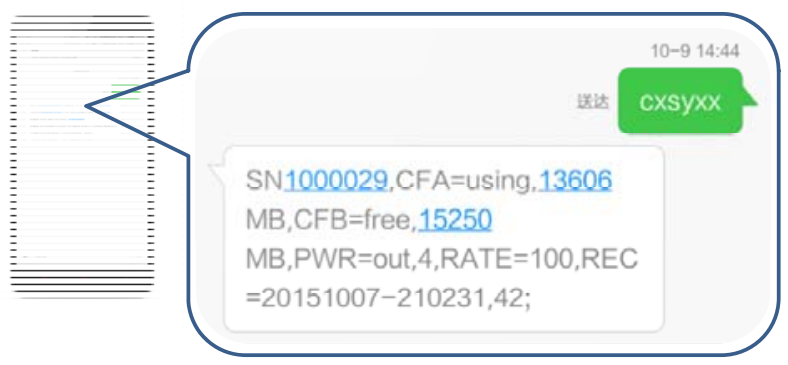

Fig. 3. Remote status monitoring interface by SMS.

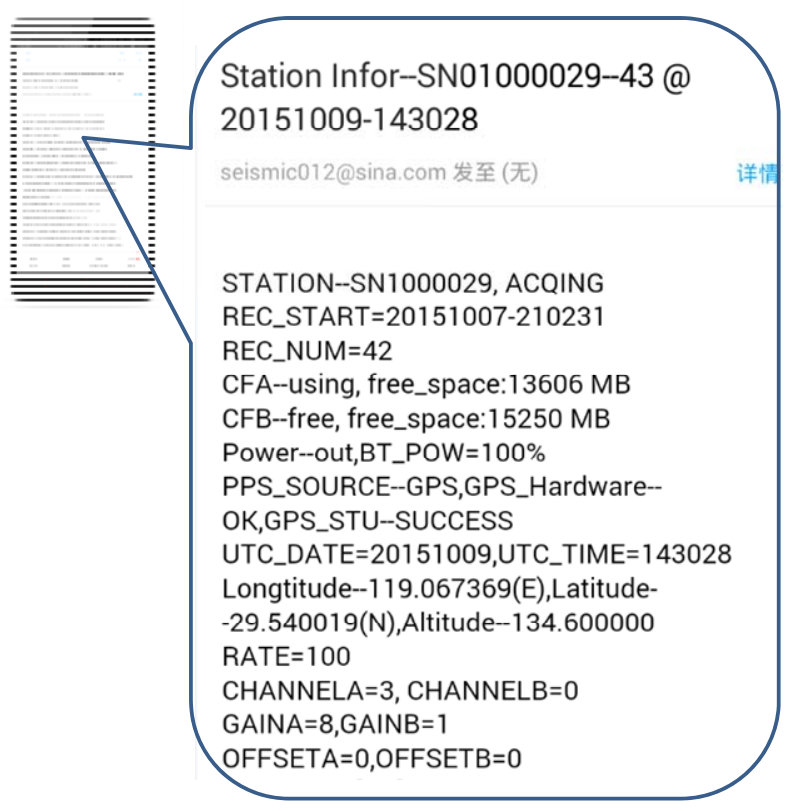

Fig. 4. Remote status monitoring interface by e-mail.

\section{CONCLUSIONS}

We designed two methods for remote status monitoring of broadband seismometer based on the mobile Internet, SMS and e-mail. In practical applications, by using our selfdeveloped broadband seismometer with remote status monitoring function, we could real-timely get status information of instruments located in remote areas in the wild. At the same time, it was stable and convenient. It greatly reduced manpower and material costs of remote status monitoring of broadband seismometer. It provided a good solution for remote status monitoring of broadband seismometer.

\section{ACKNOWLEDGMENT}

We thank other partners in our laboratory for their direct and indirect help to this research. We also thank the Editor responsible for this paper.

\section{REFERENCES}

[1] Dong Shuwen, Li Tingdong, Gao Rui, Lv Qingtian, Wei Wenbo, etc, "Progress of SinoProbe-deep exploration in China 2008-2012," Acta Geoscientia Sinica, 2013, 01:7-23.

[2] Huang Danian, Yuping, Di Qingyun, Guo ziqi, Lin Jun, etc, "Development of key instruments and technologies of deep exploration today and tomorrow,” Journal of Jilin University (Earth Science Edition), 2012, 05:1485-1496.

[3] Wu Haichao, Lin Jun, Zhang Linhang, "The study of the network communication technology applied in the seismograph,” Progress in Geophysics, 2012, 04:1822-1831.

[4] Liu Longshen, Shen Mingxia, Sun Yuwen, Lu Mingzhou, Xiong Yingjun, "Acquisition system and wireless transmission by $3 \mathrm{G}$ for farmland image based on FPGA," Transactions of The Chinese Society of Agricultural Machinery, 2011, 42(12): 186-190.

[5] Rost S, Thomas C, "Array seismology: methods and applications," Reviews of geophysics, 2002, 40(3): 2-1-2-27. 